\title{
Study of shear-thinning effect between polymer nanoparticle surfaces during shear-induced
} aggregation - Supporting Information

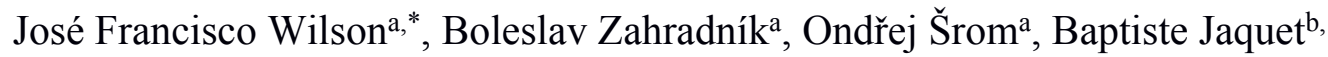 \\ †, Fatima Hassounac ${ }^{c}$, Zdeněk Hrdlička ${ }^{d}$, Juraj Kosekª, Miroslav Šoóša,
}

${ }^{a}$ University of Chemistry and Technology Prague, Department of Chemical Engineering, Technicka 5, 16628 Prague 6, Czech Republic.

${ }^{\mathrm{b}}$ ETH Zurich, Institute for Chemical and Bioengineering, Vladimir-Prelog-Weg 1-5/10, 8093

Zurich, Switzerland.

c University of Chemistry and Technology Prague, Department of Computing and Control Engineering, Technicka 3, 16628 Prague 6, Czech Republic.

${ }^{d}$ University of Chemistry and Technology Prague, Department of Polymers, Technicka 5, 16628 Prague 6, Czech Republic.

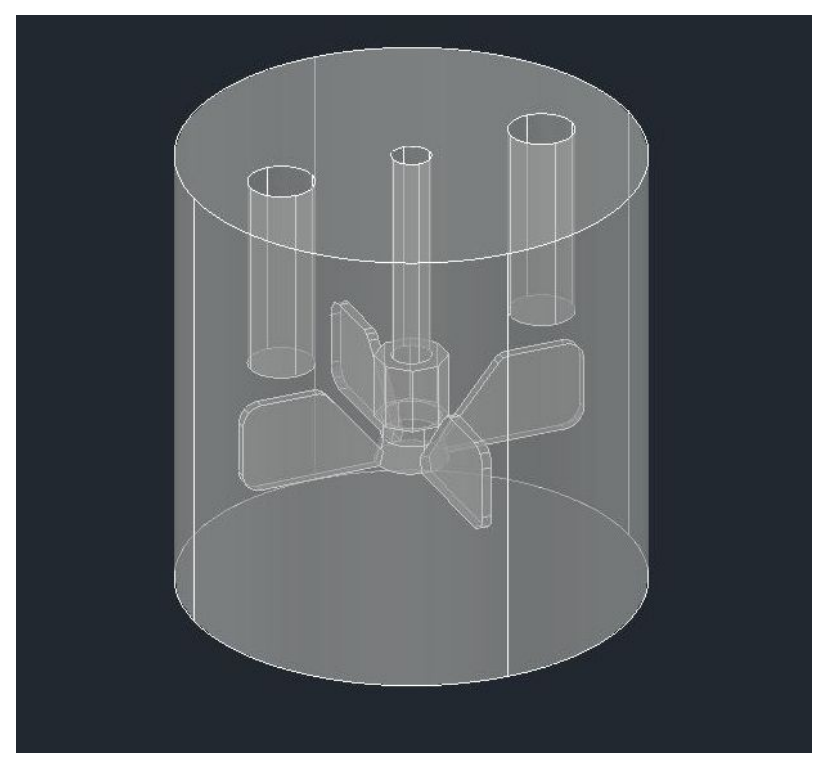

Figure S 1 - Geometry of the used stirred vessel

$\uparrow$ Current address: Givaudan Intl. AG, Kemptpark 50, 8310 Kemptthal, Switzerland

* Corresponding authors: José Francisco Wilson (wilsonj@vscht.cz), Miroslav Šoóš (miroslav.soos@vscht.cz) 

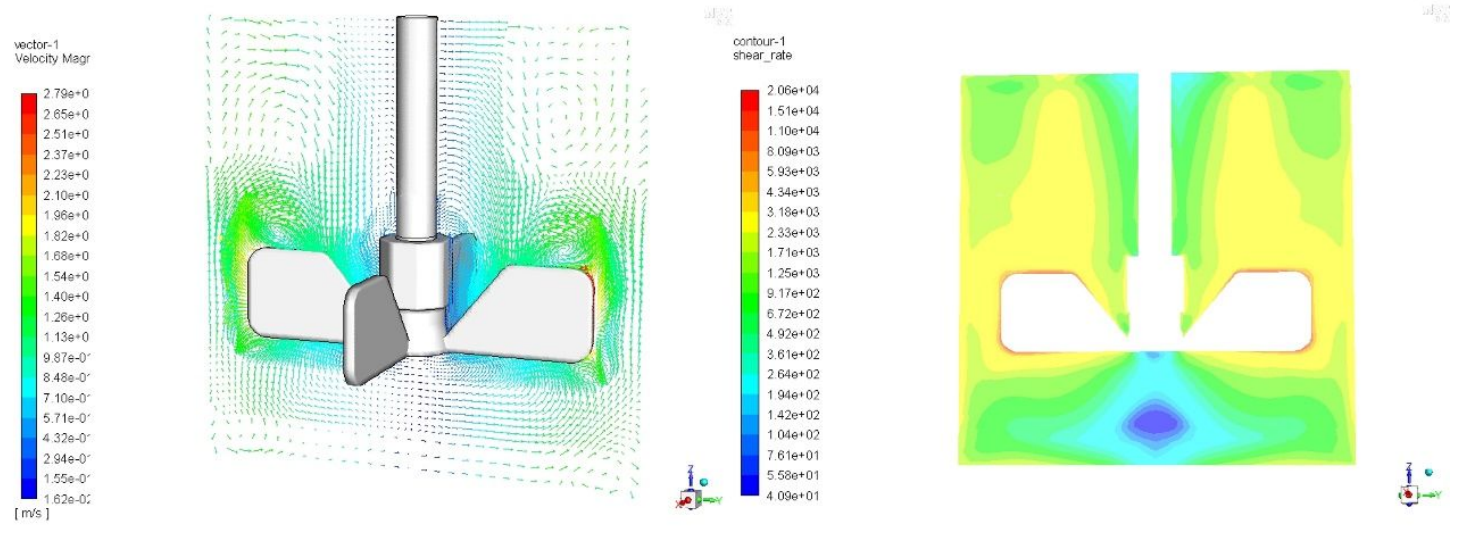

Figure S 2 (left) Velocity vectors in $\mathrm{m} / \mathrm{s}$ in used stirred vessel together with the corresponding shear rate in $1 / \mathrm{s}$ (right) plotted using log-scale. CFD model of the batch reactor calculated in Ansys Fluent calculated for temperature $25^{\circ} \mathrm{C}$ and stirring speed $800 \mathrm{rpm}$. 